\title{
Quality control of Lycium chinense and Lycium barbarum cortex (Digupi) by HPLC using kukoamines as markers
}

Yuan-Yuan $\mathrm{Li}^{1,3+}$, Rui $\mathrm{Di}^{1+}$, Wing-Leung Hsu${ }^{1}$, Ye-Qing Huang ${ }^{1}$ and Hon-Yeung Cheung ${ }^{1,2^{*}}$

\begin{abstract}
Background: Lycii Cortex (LyC), composed of Lycium chinense and Lycium barbarum cortex and having the Chinese name Digupi, is used to treat chronic diseases like cough, hypertension, and diabetes in Eastern Asia. However, chromatographic methods, such as TLC and HPLC, to determine the phytochemical composition of LyC have not been included in any official compendiums. This study aims to establish a validated HPLC method for quality control of LyC.

Methods: Kukoamines A and B (KA and KB, respectively) were selected as markers for the HPLC method. An acetic acid solution was adopted for sample extraction because it facilitated the release of kukoamines and effectively prevented their degradation. Optimal separation of the kukoamine isomers was achieved on hydrophilic ligandcoated C18 columns with a gradient elution of acetonitrile and $0.1 \%(\mathrm{v} / \mathrm{v})$ trifluoroacetic acid. The average contents and proposed contents for LyC were calculated with a $t$ test and an uncertainty test based on 16 batches of authentic samples.

Results: The method was validated with linearity $\left(r^{2}=0.9999\right.$ for both $K A$ and $\left.K B\right)$, precision (RSD $=1.29 \%$ for $K A$ and $0.57 \%$ for KB), repeatability (RSD $=1.81 \%$ for KA and $0.92 \%$ for KB), and accuracy (recovery of $90.03-102.30 \%$ for KA, and $98.49-101.67 \%$ for KB), indicating that the method could offer reliable results for quality control analysis of LyC. At the $95 \%$ confidence level, the calculated content limits were $1.45 \mathrm{mg} / \mathrm{g}$ for KA and $4.72 \mathrm{mg} / \mathrm{g}$ for KB.

Conclusion: Compared with conventional morphological identification, the HPLC method involving KA and KB contents offers precise, objective, and quantitative results for quality control of LyC.
\end{abstract}

\section{Background}

Lycii Cortex (LyC) is used to treat chronic diseases like cough, hypertension, and diabetes in Eastern Asia [1-3]. Although pharmacopeias from China, Japan, and Korea have officially stipulated the source of $\mathrm{LyC}$ as the root bark of Lycium chinense and Lycium barbarum [4-6], the herb is often misused or adulterated with other cortexes having similar appearances. Contemporary authentication of LyC relies on morphological identification [4-6]. In contrast, inspections based on characteristic

*Correspondence: cheung.honyeung@cityu.edu.hk

${ }^{\dagger}$ Yuan-Yuan Li and Rui Di contributed equally to this work

1 Research Group for Bioactive Products, Department of Biomedical

Sciences, City University of Hong Kong, Hong Kong SAR, China

Full list of author information is available at the end of the article constituents are practical and objective, because monitoring of phytochemical markers is normally not only genus-specific, but also herbal function-related [7-9].

Chromatographic techniques are commonly used for both qualitative and quantitative purposes with reliable results [10]. However, a genuine chromatographic method for LyC involving unique markers is still missing in official compendiums. Although some phytochemicals such as phenolic acids and flavonoids have been recommended as markers for quality assessment for LyC [11-13], they are neither unique nor abundant in this herb. Hence, they should not be adopted.

Recently, kukoamines A and B (KA and KB, respectively) were recommended by us as unique markers for LyC [14], because they are truly bioactivity-related, genus-specific, and content-abundant in this herb. Although the LC-MS/ 
MS method is considered to provide simultaneous and precise determination of numerous phytochemical constituents $[14,15]$, the high cost of the equipment and its operation has made this method less practical for daily quality control inspections.

This study aims to provide a validated method for quality control of $\mathrm{LyC}$ with reference to $\mathrm{KA}$ and $\mathrm{KB}$. Based on the developed method, multiple batches of LyC were investigated and the content limits were statistically calculated.

\section{Methods}

\section{Reagents}

Methanol and acetonitrile (HPLC grade) were purchased from Labscan Asia (Thailand). Trifluoroacetic acid (TFA) and acetic acid (both HPLC grade) were purchased from Fluka (Switzerland). All other chemicals were of analytical grade. Water was prepared using a Millipore MilliQPlus system (Millipore, USA). KA and KB (Fig. 1) were extracted and purified by our group (purity: higher than 98\%) [16].

\section{Plant materials}

Dried root bark samples of $L$. chinense and L. barbarum were collected from different regions in China and a local market in Hong Kong (Table 1). At least $2 \mathrm{~kg}$ of each bark was collected. The species of the plants were identified by Dr. Zhi-Feng Zhang (Ethnic Pharmaceutical Institute, Southwest University for Nationalities). The voucher specimens (DGP-JXXS-001, DGP-HBQL-001, and DGPJXJJ-001) were stored in the Department of Health, Hong Kong Government SAR.

LyC sliced samples, with the commercial names Hong Digupi (RLyC) and Bai Digupi (WLyC), were provided by herb stores from the Hehuachi herbal market in Chengdu
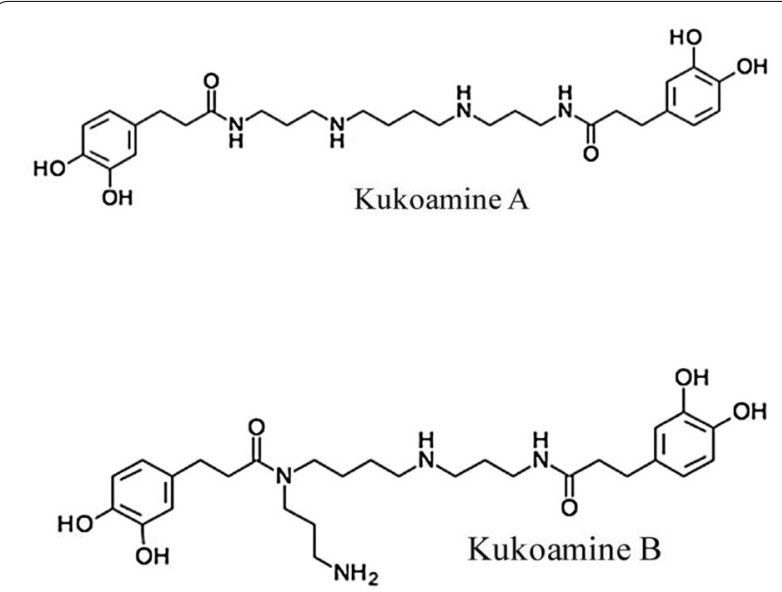

Fig. 1 Structures of kukoamines $A$ and $B$
(Sichuan Province, China). These samples had unclear sources, and were classified based on traditional experience. LyC extracts (Nos. 01-03) were bought from herbal extract companies in Sichuan, Shanxi, and Hubei. These extracts were claimed to be extracted from $\mathrm{LyC}$.

\section{Chromatographic conditions}

The HPLC analysis was performed on an Agilent 1260 system (Agilent, USA) equipped with an on-line degasser (G1322A; Agilent, USA), a binary bump (G1312C; Agilent, USA), an autosampler (G1329B; Agilent, USA), a column oven (G1316A; Agilent, USA), and a diode array detector (G1315D; Agilent, USA). A Zorbax C18 SB-AQ column ( $250 \mathrm{~mm} \times 4.6 \mathrm{~mm}$ i.d., $5 \mu \mathrm{m}$; Agilent, USA) was used for the separation. A mobile phase consisting of $0.1 \%$ TFA (A) and acetonitrile (B) was applied for the separation with the following gradient program: 0-15 min, $12-16 \% \mathrm{~B} ; 15-35 \mathrm{~min}, 16-22 \% \mathrm{~B}$. The flow rate was $1.0 \mathrm{~mL} / \mathrm{min}$ and the column oven was set at $40{ }^{\circ} \mathrm{C}$. The detection wavelength was $280 \mathrm{~nm}$.

\section{Sample preparation}

Each LyC sample was cut into small pieces and pulverized into a powder (passed through a 200-mesh sieve). The herbal powder $(0.5 \mathrm{~g})$ was accurately weighed. The extraction solvent was a $50 \%$ methanol aqueous solution containing $0.5 \%$ acetic acid (v/v). After addition of $20 \mathrm{~mL}$ of the extraction solvent, the mixture was sonicated in an ultrasonic bath (100 W, AC- $120 \mathrm{H}$; MRC, Germany) for $30 \mathrm{~min}$ and then centrifuged at $2880 \times g$ for $10 \mathrm{~min}$ (Allegra X-15R; Beckman Coulter, USA). The supernatant was transferred into a $50-\mathrm{mL}$ volumetric flask and the pellet was re-extracted with another $20 \mathrm{~mL}$ of extraction solvent for $30 \mathrm{~min}$. After centrifugation, the extracted solution was transferred to the same volumetric flask. Next, $10 \mathrm{~mL}$ of extraction solvent was used to wash the tube, and the washing solution was also added to the volumetric flask and filled to the mark. The prepared sample solution was filtered through a $0.22-\mu \mathrm{m}$ pore-size filter before injection.

\section{Method validation}

Calibration curves for KA and KB were constructed by plotting the peak area versus the standard concentration. The calibration curves ranged from $3.91-250.00 \mathrm{mg} / \mathrm{L}$ for KA and 4.12-263.50 mg/L for KB. The method precision was measured by the relative standard deviation (RSD; \%) of the peak areas determined for six successive injections of the standard solution. The repeatability test was measured by injection of five replicate samples for the proposed sample preparation and determination methods. The repeatability was calculated by the RSD 
Table 1 Production areas and species of Lycii Cortex samples and the contents of kukoamines A and B

\begin{tabular}{|c|c|c|c|c|c|}
\hline Batch no. & Species & Origin & Collection year & Content of $K A^{a}(\mathrm{mg} / \mathrm{g})$ & Content of $\mathrm{KB}^{\mathrm{a}}(\mathrm{mg} / \mathrm{g})$ \\
\hline LC 1 & L. chinense & Jiangxi & 2011 & $9.65 \pm 0.26$ & $5.03 \pm 0.13$ \\
\hline LC 2 & L. chinense & Jiangxi & 2011 & $4.38 \pm 0.27$ & $13.27 \pm 0.04$ \\
\hline LC 3 & L. chinense & Jiangxi & 2011 & $3.33 \pm 0.18$ & $13.50 \pm 0.18$ \\
\hline LC 4 & L. chinense & Jiangxi & 2011 & $1.82 \pm 0.13$ & $3.39 \pm 0.22$ \\
\hline LC 5 & L. chinense & Gansu & 2011 & $3.83 \pm 0.03$ & $13.29 \pm 0.14$ \\
\hline LC 6 & L.chinense & Shangxi & 2011 & $6.34 \pm 0.11$ & $22.08 \pm 0.11$ \\
\hline LC 7 & L.chinense & Neimenggu & 2011 & $1.13 \pm 0.02$ & $3.49 \pm 0.02$ \\
\hline LC 8 & L. chinense & Neimenggu & 2011 & $4.87 \pm 0.04$ & $14.27 \pm 0.27$ \\
\hline LC 9 & L. barbarum & Ningxia & 2011 & $1.86 \pm 0.09$ & $6.80 \pm 0.18$ \\
\hline LC 10 & L. barbarum & Ningxia & 2011 & $1.93 \pm 0.02$ & $6.65 \pm 0.01$ \\
\hline LC 11 & L. barbarum & Ningxia & 2011 & $4.06 \pm 0.23$ & $16.88 \pm 0.38$ \\
\hline LC 12 & L. barbarum & Ningxia & 2011 & $1.47 \pm 0.01$ & $4.76 \pm 0.23$ \\
\hline LC 13 & L. barbarum & Ningxia & 2011 & $3.96 \pm 0.29$ & $19.02 \pm 0.11$ \\
\hline LC 14 & L. chinense & Hong Kong & 2011 & $1.29 \pm 0.03$ & $3.47 \pm 0.15$ \\
\hline LC 15 & L.chinense & Hong Kong & 2012 & $4.25 \pm 0.04$ & $18.45 \pm 0.60$ \\
\hline LC 16 & L.chinense & Hong Kong & 2012 & $1.75 \pm 0.02$ & $7.60 \pm 0.02$ \\
\hline
\end{tabular}

a The content was converted to the dry basis. The experiments for each sample were performed in triplicate $(n=3)$, and the results are presented as the mean \pm standard deviation

of the contents obtained from the five replicate samples [17]. The sample recovery was assessed by adding known amounts of individual standards into an accurately weighted sample. Three concentrations were investigated and triplicate measurements were performed for each concentration. Recovery was calculated with the following equation [16]:

Recovery $(\%)=100 \times($ amount found - original amount $) /$ amount spiked

The method detection limit (MDL) and limit of quantitation (LOQ) were determined according to the guidelines in Validation of Analytical Procedures: Methodology (Q2B) [18].

\section{Statistical analysis}

The contents of samples were presented as the mean \pm standard deviation. The proposed content limit for each analyte was calculated according to the contents from multiple batches with consideration of the standard uncertainty of the precision, bias, purity of reference substance, and water content (the calculation equation is shown in Additional file 1). The calculation was based on the ISO "Guide to the Expression of Uncertainty in Measurement" [19], EURACHEM/CITAC document "Quantifying Uncertainty in Analytical Measurement" [20], and LGC document "Development and Harmonization of Measurement Uncertainty Principles" [21]. The mean differences for KA and KB in the two species of L. chinense and $L$. barbarum were evaluated by analysis of variance
(ANOVA). If the $P$ value was less than 0.05 , a follow-up post hoc test was conducted.

\section{Results and discussion}

Establishment of HPLC conditions for determination of kukoamines

Selection of column, mobile phase, and detection wavelength $\mathrm{KA}$ and $\mathrm{KB}$ are water-soluble spermine alkaloids with multiple amino and amide groups [22, 23] that cause peak tailing on the $\mathrm{C}_{18}$ packing material. Three kinds of $\mathrm{C}_{18}$ columns with special coatings on the packing surface, i.e., Zorbax $\mathrm{C}_{18}$ BDS, Zorbax $\mathrm{C}_{18}$ Extended, and Zorbax $\mathrm{C}_{18}$ SB-AQ (all from Agilent, USA), were compared for the analyte retention behaviors. The kukoamines showed better retention on the Zorbax $\mathrm{C}_{18} \mathrm{SB}-\mathrm{AQ}$ column, owing to the better affinity of the extremely water-soluble analytes for the hydrophilic packing surface.

The influence of acids on the separation was investigated (Table 2). Within the $\mathrm{pH}$ range of 2.0-2.5, TFA was superior to phosphoric acid and formic acid in obtaining satisfactory peak resolution and symmetry. Furthermore, the peak sequence of KA and KB observed with TFA was the opposite to that observed with the other two acids. This difference was caused by the formation of ion pairs [24], which greatly altered the interaction between the analytes and the packing surface. Owing to the better separation performance, TFA was chosen as the acid for the mobile phase. The detection wavelength chosen for the kukoamines was $280 \mathrm{~nm}$, based on the characteristic UV absorption of the $o$-dihydrocaffeoyl groups in the analytes. 
Table 2 Influence of acids on chromatographic behaviors of kukoamines

\begin{tabular}{|c|c|c|c|c|c|}
\hline Aqueous phase $^{a}(v / v)$ & Analytes & Retention time ( $\mathrm{min}$ ) & Plates $^{\mathbf{b}}$ & $\mathrm{R}^{\mathbf{c}}$ & $\mathrm{T}^{\mathrm{d}}$ \\
\hline \multirow[t]{2}{*}{ Water } & KA & 15.3 & 126 & 0.8 & 2.30 \\
\hline & KB & 20.9 & 52 & & 4.40 \\
\hline \multirow[t]{2}{*}{$0.2 \%$ Formic acid (v:v) pH 2.5} & KA & 34.6 & 4557 & 1.0 & 3.02 \\
\hline & KB & 37.8 & 4632 & & 3.06 \\
\hline \multirow[t]{2}{*}{ 0.1\% Phosphate acid (v:v) pH 2.0} & KA & 35.1 & 3954 & 0.7 & 2.01 \\
\hline & KB & 37.9 & 3249 & & 2.52 \\
\hline \multirow[t]{2}{*}{ 0.1\% Trifluoroacetic acid (v:v) pH 2.0} & KA & 47.3 & 10,091 & 2.6 & 1.10 \\
\hline & $\mathrm{KB}$ & 37.1 & 10,887 & & 1.12 \\
\hline
\end{tabular}

a The exp eriment was conducted on an Agilent Zorbax $\mathrm{C}_{18} \mathrm{SB}-\mathrm{AQ}$ column $(250 \mathrm{~mm} \times 4.6 \mathrm{~mm}$ i.d., $5 \mu \mathrm{m})$ with fixed proportions of acetonitrile and aqueous phase (16:84). The aqueous phase was investigated with different acids

b The number of theoretical plates was calculated as $N=5.54\left(t_{R} / W_{h / 2}\right)^{2}$, where $t_{R}$ is the retention time and $W_{h / 2}$ is the peak width at half height

${ }^{c}$ The resolution $R$ refers to the resolution between $K A$ and $K B$. It was calculated as $R=2\left(t_{A}-t_{B}\right) /\left(W_{A}+W_{B}\right)$, where $t_{A}$ and $t_{B}$ are the retention times and $W_{A}$ and $W_{B}$ are the peak widths

$\mathrm{d}$ The tailing factor $\mathrm{T}$ was calculated as $\mathrm{T}=\mathrm{W}_{0.05 \mathrm{~h}} / 2 \mathrm{f}$, where $\mathrm{W}_{0.05 \mathrm{~h}}$ is the peak width at $5 \%$ peak height and $\mathrm{f}$ is the width start point at $5 \%$ peak height to the time of the maximum point

\section{Influence of $\mathrm{pH}$ on the stability of the kukoamines}

A stability test for the analytes was performed, and the results are shown in Fig. 2. KA and $\mathrm{KB}$ were not stable in neutral solution $(\mathrm{pH}=7)$, as they were found to decrease by about $30 \%$ after exposure in air for $21 \mathrm{~h}$. This change might arise through oxidation of the $o$-dihydrocaffeoyl moieties into quinones [25]. In contrast, it was found that when the $\mathrm{pH}$ was adjusted to $2.5-3$ by acetic acid, KA and KB remained constant within the investigated period. This protective effect can be explained by the formation of hydrogen bonds between the phenolic hydroxyl groups and the solvent molecules in the acidic solution, which greatly protected the phenolic hydroxyl groups from oxidization [26]. Therefore, an acidic solution $(0.5 \%$ acetic acid aqueous solution, v/v) was preferred for both the standard and sample solutions.

\section{Optimization of kukoamine extraction procedures}

Key factors influencing the extraction efficiency, specifically the extraction solvent and number of extraction rounds, were optimized to completely extract the analytes for analysis. The 50 and $80 \%$ methanol solutions $(\mathrm{v} / \mathrm{v})$ showed similar performances, which were much higher than those for the other concentrations (Fig. 3a). The optimal concentration of methanol for extraction was found to be $50 \%(\mathrm{v} / \mathrm{v})$ from the aspect of ecofriendliness. Figure $3 b$ shows the effects of acids on the extraction efficiency. The acidic solution was about onefold more potent than the neutral solution for kukoamine extraction. Therefore, $0.5 \%$ acetic acid (v/v) was adopted, because it facilitated the release of alkaloids from the powder to the solvent. The influence of the number of extraction rounds was also examined (Fig. 3c). When samples were sonicated twice, almost all of the kukoamines were completely extracted. Therefore, two extractions were chosen for our extraction procedure.

\section{Method validation}

The established method was validated for its detection limit (MDL), quantification limit (LOQ), linearity, precision, reproducibility, and recovery. As shown in Table 3, the high correlation coefficient $\left(\mathrm{r}^{2}=0.9999 ; P<0.05\right)$ indicated good linearity between the concentrations of kukoamines and their peak areas. The MDL and LOQ were derived from the responses of the analytes to $\mathrm{UV}$, and were found to be 1.76 and $8.78 \mu \mathrm{g} / \mathrm{mL}$ for kukoamine A, and 1.86 and $9.29 \mu \mathrm{g} / \mathrm{mL}$ for kukoamine $\mathrm{B}$, respectively. The RSD (\%) in the recovery, precision, and reproducibility tests were all less than $2 \%$. The good recovery obtained further suggested that the method was accurate and reliable.

\section{Quantification results and proposed contents}

The validated method was applied to investigation of the LyC samples from different locations, which covered most of the production regions in China. The post-harvest treatment of the herb strictly followed the procedures described in the professional compendiums to ensure quality consistency [4, 27]. A typical chromatogram of LyC and the overlay chromatograms for multiple batches are shown in Fig. 4a, b. The chromatographic patterns of all samples, regardless of whether they were L. barbarum or L. chinense, were highly consistent, although the abundance of some peaks varied remarkably. With the proposed chromatographic methods, the common peaks (1-6) in Fig. 4 b could be used as the fingerprinting features for $\mathrm{LyC}$ authentication. The contents of $\mathrm{KA}$ and $\mathrm{KB}$ in the investigated $\mathrm{LyC}$ samples were in the ranges of $1.13-9.65$ and $3.39-22.08 \mathrm{mg} / \mathrm{g}$, respectively 


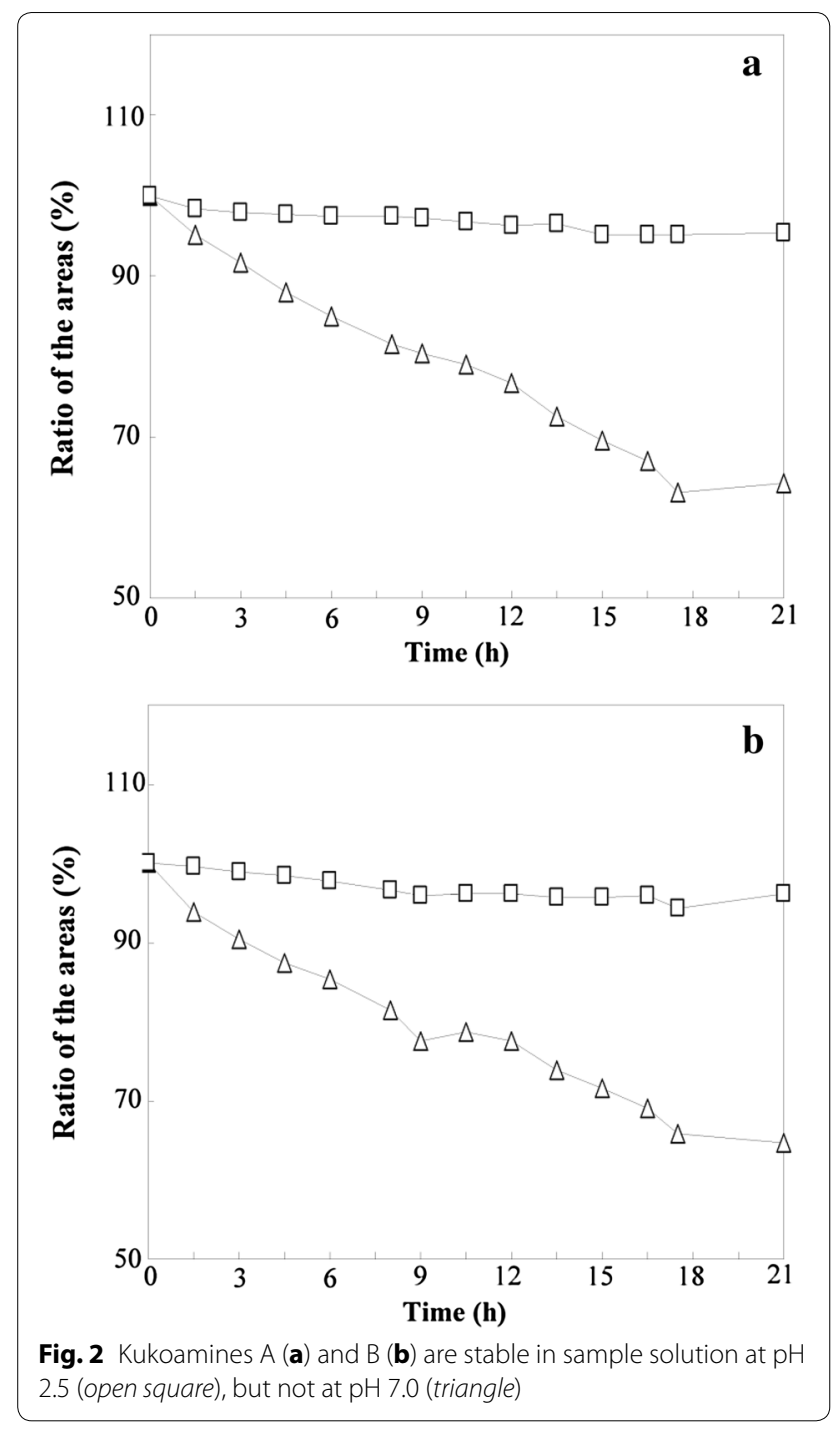

(Table 1). In general, KB was more abundant than KA in the LyC samples.

The LyC samples from $L$. chinense and L. barbarum did not differ significantly from each other in their kukoamine contents when compared by one-way ANOVA (Additional file 1). These findings demonstrated that both plants can produce $\mathrm{LyC}$ with equivalent quality.

Geography, soil, and climate can cause fluctuations in herbal constituents [28-30]. Nevertheless, the secondary metabolites in LyC, KA and $\mathrm{KB}$, typically fitted a normal distribution model [19-21]. Therefore, we estimated the content ranges of $\mathrm{KA}$ and $\mathrm{KB}$ for the LyC population using data from the collected representative samples by statistical inference [19-21] (Additional file 2). At the 95\% confidence level, the calculated content limits were $1.45 \mathrm{mg} / \mathrm{g}$ for KA and $4.72 \mathrm{mg} / \mathrm{g}$ for KB (Table 4). These content limits could be used as reference values to judge the quality of LyC samples (Table 4).

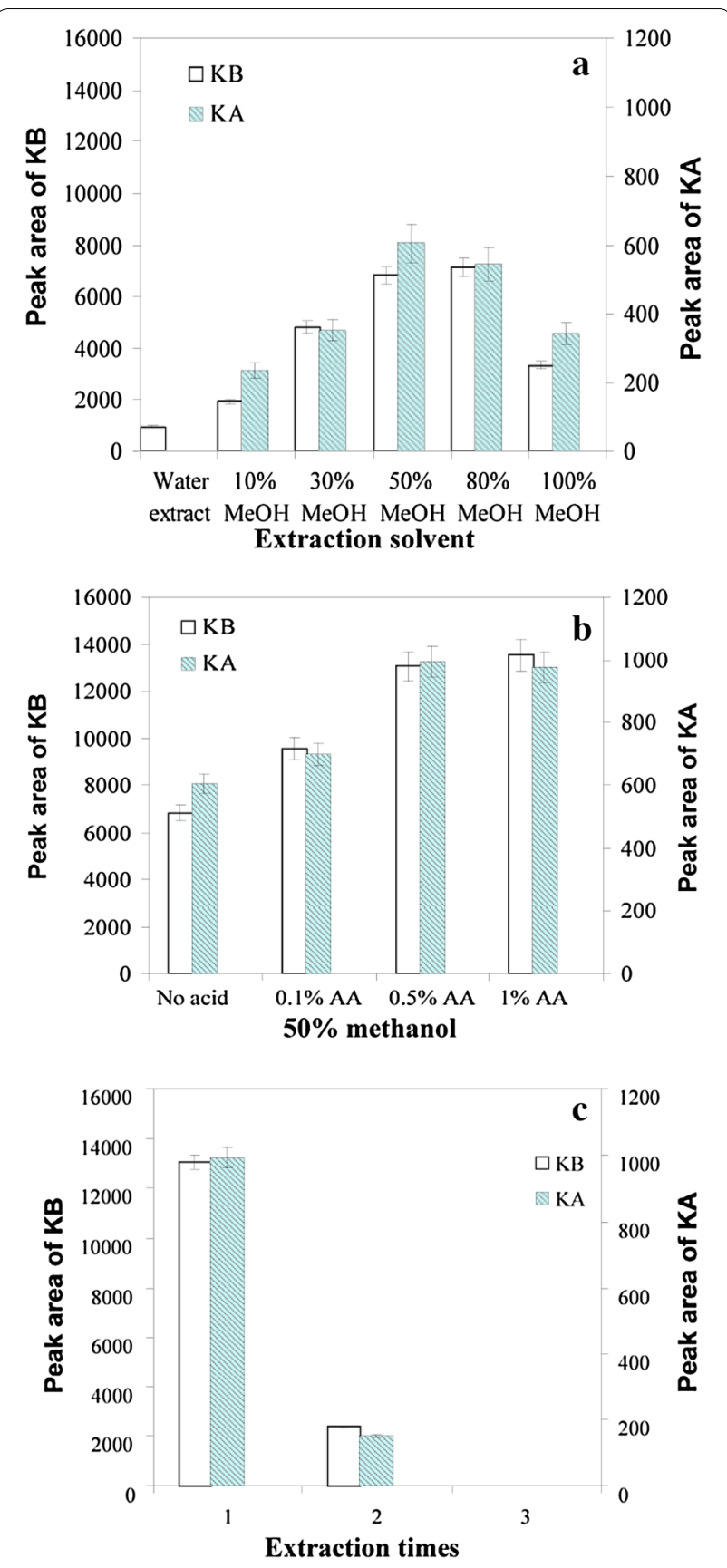

Fig. 3 Influence of methanol concentration (a), acetic acid concentration (b), and number of extraction rounds (c) on the extraction efficiency of kukoamines in LyC. The experiments for each condition were performed in triplicate $(n=3)$ and the results are presented as the mean \pm standard deviation

\section{Application of the HPLC method for quality control of LyC}

Five batches of unauthenticated samples, which were claimed to be LyC sliced pieces and extracts, were tested by the established method and then judged by the calculated quality criteria. As shown in Fig. 5, only the crude material called "Hong Digupi" and the extract powder 
Table 3 Linearity, precision, repeatability, recovery, MDL, and LOQ of the established method

\begin{tabular}{|c|c|c|}
\hline Parameter & Kukoamine A & Kukoamine B \\
\hline Linearity (12 points) & $3.91-250.00 \mathrm{mg} / \mathrm{L} ; \mathrm{r}^{2}=0.9999^{\mathrm{a}}$ & $4.12-263.50 \mathrm{mg} / \mathrm{L} ; \mathrm{r}^{2}=0.9999^{\mathrm{a}}$ \\
\hline Precision (RSD) $)^{\mathrm{b}}, \%$ & 1.29 & 0.57 \\
\hline Repeatability (RSD)c, \% & 1.81 & 0.92 \\
\hline Mean recovery $(n=5)^{d}$ & $\begin{array}{l}95.89 \% \\
90.03-102.30 \%\end{array}$ & $\begin{array}{l}100.32 \% \\
98.49-101.67 \%\end{array}$ \\
\hline $\mathrm{MDL}(\mu \mathrm{g} / \mathrm{mL})$ & 1.76 & 1.86 \\
\hline LOQ $(\mu \mathrm{g} / \mathrm{mL})$ & 8.78 & 9.29 \\
\hline
\end{tabular}

MDL method detection limit, $L O Q$ limit of quantification

a $\mathrm{r}^{2}$, square of correlation coefficient for the linear regression $(P<0.05)$

b Precision was tested based on six injections of a standard solution with $62.50 \mu \mathrm{g} / \mathrm{mL}$ kukoamine $\mathrm{A}$ and $131.75 \mu \mathrm{g} / \mathrm{mL} \mathrm{kukoamine} B$. RSD (\%) $=100 \times \mathrm{SD} / \mathrm{mean}$

c Repeatability was tested on five replicate samples

${ }^{d}$ Recovery $(\%)=100 \times$ (amount found - original amount)/amount spiked. The results were presented as the mean recovery and recovery range. The amounts of spiked components for the recovery test were $10.4,20.8$, and $41.6 \mathrm{mg}$ for $\mathrm{KB}$, and $1.5,3.0$, and $4.5 \mathrm{mg}$ for $\mathrm{KA}$
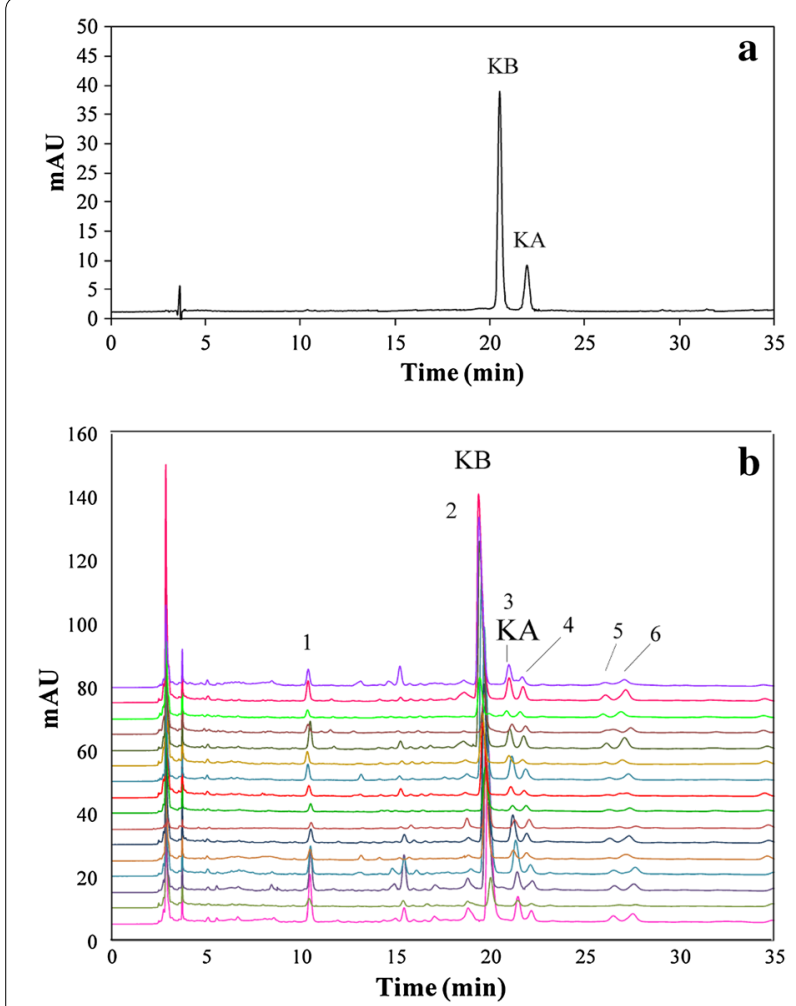

Fig. 4 HPLC chromatogram of mixed standards (a) and fingerprint profiles of LyC (b) from multiple batches. The numbers $1-6$ in $\mathbf{b}$ indicate the common fingerprinting peaks for identification

from Sichuan presented the proper amounts of kukoamines, while other samples did not contain any kukoamines. The "Bai Digupi" on the market was actually not from the root bark of either L. chinense or L. barbarum. The lack of kukoamines in the investigated LyC extract powder might be caused by an inappropriate extraction
Table 4 Mean contents and proposed content limits for kukoamines A and B in Lycii Cortex

\begin{tabular}{lll}
\hline Parameters & KA & KB \\
\hline $\mathrm{Ca}^{\mathrm{a}}$ & 3.49 & 10.75 \\
$\mathrm{SD}^{\mathrm{b}}$ & 2.51 & 6.31 \\
$\mathrm{RSD}^{\mathrm{c}}$ & 71.91 & 58.73 \\
$\mathrm{n}^{\mathrm{d}}$ & 16 & \\
$\mathrm{t}_{\text {critical }}^{\mathrm{e}}$ & 2.131 & \\
$\mathrm{uc}^{\mathrm{C}}(\mathrm{C})^{\mathrm{f}}$ & 0.46 & 2.67 \\
Calculated limit $^{\mathrm{g}}$ & 1.45 & 4.72 \\
Outlier $^{\text {h }}$ & 0 & 0 \\
${\text { Failing rate }(\%)^{\mathrm{i}}}^{\text {a }}$ & 12.50 & 18.75
\end{tabular}

${ }^{a} \mathrm{Ca}$, average content of klkukoamines in samples $(\mathrm{mg} / \mathrm{g})$

${ }^{b} \mathrm{SD}$, standard deviation of the contents in all samples

c $\mathrm{RSD}(\%)=100 \times \mathrm{SD} /$ mean

${ }^{d} \mathrm{n}$, sample size

e $t_{\text {critical }}$ critical value for a 95\% confidence interval when the sample size was 16

f uc (C), combined standard uncertainty of the kukoamine B content in the sample $(\mathrm{mg} / \mathrm{g})$. The calculation is shown in the supplementary data section

${ }^{9}$ Calculated limit for kukoamines in the sample. The calculation is shown in the supplementary data section

${ }^{\mathrm{h}}$ Number of outlier samples. The data are referenced to Table 1

i Percentage of samples with kukoamine contents outside the calculated limits

method that damaged the major bioactive compounds, or extraction from the wrong source plants.

Compared with the conventional morphological identification, the proposed HPLC method for monitoring the characteristic constituents $\mathrm{KA}$ and $\mathrm{KB}$ provides a simple and universal way to measure the quality of LyC samples. The method and calculated content limits presented in this article could support amendments to the quality standards for LyC in official compendiums. 


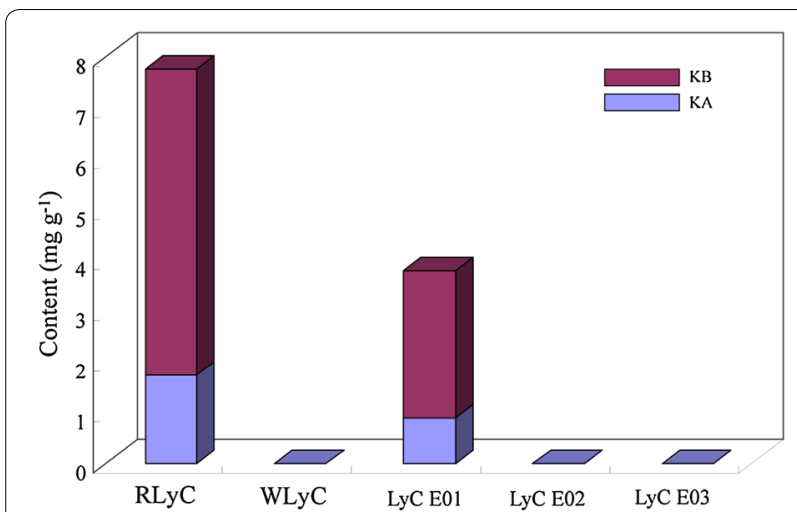

Fig. 5 Quality assessment of LyC sliced samples and extracts from markets. RLyC Hong Digupi, WLyC Bai Digupi, LyC E 01-03 LyC extracts from manufacturers $01-03$

\section{Conclusion}

The developed method could be applied for quality inspection of LyC in the food and pharmaceutical manufacturing industries.

\section{Additional files}

Additional file 1: Table S1. Comparison of LyCs from $L$. chinensis and $L$. barbarum in terms of kukoamines $A$ and B using ANOVA test.

Additional file 2. Equations for calculation of the content limits of kukoamines.

\section{Abbreviations}

LyC: Lycii Cortex; KA: kukoamine A; KB: kukoamine B; HPLC: high performance liquid chromatography; LOQ: limit of quantification; MDL: method detection limit; RSD: relative standard deviation; TFA: trifluoroacetic acid.

\section{Authors' contributions}

LYY and DR conceived and designed the study. CHY secured financial support and oversaw the whole study. DR, LYY, and HWL performed the experiments. HYQ performed the statistical analysis. LYY and DR drafted the manuscript and $\mathrm{CHY}$ revised it. All authors read and approved the final manuscript.

\section{Author details}

${ }^{1}$ Research Group for Bioactive Products, Department of Biomedical Sciences, City University of Hong Kong, Hong Kong SAR, China. ${ }^{2}$ Key Laboratory of Biochip Technology, Shenzhen Biotech and Health Centre, City University of Hong Kong, Shenzhen 518057, China. ${ }^{3}$ Present Address: System and Translational Science Center, RTI International, Research Triangle Park, NC 27709, USA.

\section{Acknowledgements}

The authors also extend their gratitude to Mr. William T. Mahan for language editing.

\section{Competing interests}

The authors declare that they have no competing interests.

\section{Funding}

Hong Kong Chinese Materia Medica Standards, Department of Health, Hong Kong Government, SAR, China, for the CityU project (No. 9210029).
Received: 2 November 2016 Accepted: 6 December 2016

Published online: 09 January 2017

\section{References}

1. Ministry of Health of RP China. Document for further management of materials for manufacture functional food. 2002. http://www.moh.gov. cn/zhuzhan/wsbmgz/201304/e33435ce0d894051b15490aa3219cdc4. shtml.

2. Yao X, Peng Y, Xu LJ. Phytochemical and biological studies of Lycium medicinal plants. Chem Biodivers. 2011;8:976-1010.

3. Potterat O. Goji (Lycium barbarum and L. chinese): phytochemistry, pharmacology and safety in the perspective of traditional uses and recent popularity. Planta Med. 2010;76:7-19.

4. Committee of National Pharmacopoeia. Pharmacopoeia of the People's Republic of China. Beijing: Chemical Industry Press; 2010. p. 115.

5. Ministry of Health. Labor and welfare: The Japanese pharmacopoeia. Tokyo: Pharmaceuticals and Medical Devices Agency; 2012. p. 1686.

6. Korea Food and Drug Administration. The Korean herbal pharmacopoeia Seoul: Ministry of health family welfare of South Korea; 2007. p. 1058-9.

7. Liang XM, Jin Y, Wang YP. Qualitative and quantitative analysis in quality control of traditional Chinese medicines. J Chromatogr A. 2009;1216:2033-44

8. Lau AJ, Woo SO, Koh HL. Analysis of saponins in raw and steamed Panax notoginseng using high-performance liquid chromatography with diode array detection. J Chromatogr A. 2003;1011:77-87.

9. Shi Z, He J, Yao T. Simultaneous determination of cryptotanshinnone, tanshinone I and tanshinone IIA in traditional Chinese medicinal preparations containing Radix salvia miltiorrhiza by HPLC. J Pharm Biomed Anal. 2005;37:481-6.

10. Wu HF, Guo J, Chen SL. Recent developments in qualitative and quantitative analysis of photochemical constituents and their metabolites using liquid chromatography-mass spectrometry. J Pharm Biomed Anal. 2012;72:267-91.

11. Chu QC, Fu L, Lin M. Study on bioactive ingredients in Cortex Lycii by capillary zone electrophoresis with amperometric detection. Chin J Anal Chem. 2005;33:1611-4.

12. Li K, Chen XH, Bi KS. Determination of vanillic acid in Cortex Lycii by RPHPLC. Chin Tradit Herbal Drugs. 2005;36:446-7.

13. Li K, Li Q, Yuan JS. Determination of scopoletin in Cortex Lycii by RP-HPLC. Chin Pharm J. 2005:40:1741-2.

14. Li YY, Di R, Baibado JT. Identification of kukoamines as the novel markers for quality assessment of Lycii Cortex. Food Res Int. 2014;35:373-80.

15. Zhang JX, Guan SH, Yang M. Simultaneous determination of 24 constituents in Cortex Lycii using high-performance liquid chromatography-triple quadrupole mass spectrometry. J Pharm Biomed Anal. 2013;77:63-70.

16. Cheung HY, Li YY, Di R, Process for isolating kukoamine. USP $9012687 B 2$.

17. US Environmental Protection Agency. Technique support document for the assessment of detection and quantitation approaches. EPA821-R-03-005, Washington D.C. 2003.

18. ICH, H. T. G. Validation of Analytical Procedure: Methodology (Q2B). International conference on harmonization. USA; 1997.

19. International Organization for the Standardization: ISO/IEC Guide 98-3 uncertainty of measurement-part 3: quide to the expression of uncertainty in measurement (GUM: 1995). Geneva; 2008

20. Ellison SLR, Roesslein M, Williams A. EURACHEM/CITAC Guide: quantifying uncertainty in analytical measurement, 2nd ed. 2000. http://www.vtt.fi/ ket/eurachem/quam2.pdf.

21. Barwick VJ, Ellison SLR. VAM Project 3.2.1 Development and Harmonisation of Measurement Uncertainty Principles Part (d): Protocol for Uncertainty Evaluation from Validation Data, Version 5.1, LGC (Teddington) Limited. 2000. http://blpd.dss.go.th/knowledge_el/NAM_uncertainty-0452. pdf.

22. Parr A, Mellon FA, Colquhoun IJ. Dihydrocaffeoyl polyamines (kukoamine and allies) in potato (Solanum tuberosum) tuber detected during metabolite profiling. J Agr Food Chem. 2005;53:5461-6.

23. Rogoza LN, Salakhutdinov NF, Tolstikov GA. Plant alkaloids of the polymethyleneamine series. Russ Chem Rev. 2005;74:381-96. 
24. Cai B, Li J. Evaluation of trifluoroacetic acid as an ion-pair reagent in the separation of small ionizable molecules by reversed-phase liquid chromatography. Anal Chim Acta. 1999;399:249-58.

25. Li YY, Wang H, Zhao C, Huang Y, Tang X, Cheung HY. Inentification and characterization of kukoamine metabolites by multiple ion monitoring triggered enhanced product ion scan method with a triple-quadruple linear ion trap mass spectrometer. J Agric Food Chem. 2015;63:10785-90.

26. Sjödin M, Irebo T, Utas JE, Lind J, Merényi G, Akermark B, Hammarström L. Kinetic effects of hydrogen bonds on proton-coupled electron transfer from phenols. J Am Chem Soc. 2006;128:13076-83.

27. Wagner H, Bauer R, Melchart D. Chromatographic fingerprint analysis of herbal medicines: thin-layer and high performance liquid chromatography of Chinese drugs (vol 1). New York: Springer; 2011. p. 509-19.
28. Yi LZ, Yuan DL, Liang YZ. Quality control and discrimination of Percarpium Citri Reticulatae and Pericarpium Citri Reticulatae Viride based on high-performance liquid chromatographic fingerprints and multivariate statistical analysis. Anal Chim Acta. 2007;588:207-15.

29. Liang YZ, Xie PS, Chan K. Quality control of herbal medicines. J Chromatogr A. 2004;812:53-70.

30. Zhao ZZ, Yuen JPS, Wu J. A system study on confused species of Chinese Materia Medica in the Hong Kong market. Ann Acad Med Singapore. 2006:35:764-9.

\section{Submit your next manuscript to BioMed Central and we will help you at every step:}

- We accept pre-submission inquiries

- Our selector tool helps you to find the most relevant journal

- We provide round the clock customer support

- Convenient online submission

- Thorough peer review

- Inclusion in PubMed and all major indexing services

- Maximum visibility for your research

Submit your manuscript at www.biomedcentral com/submit 